Objectives Stroke is the third most common cause of death in developed countries, exceeded only by coronary heart disease and cancer, but there is still little knowledge on occupational risk factors. A systematic critical review was performed to assess the strength of evidence for causal associations between workrelated psychosocial risk factors, shift work and stroke.

Method Literature on stroke incidence or mortality and occupational factors published up to 2012 was identified from Medline and other relevant databases. The 4471 abstracts were evaluated independently by two reviewers. Six studies relevant to shift work and eight studies (among them four cohorts from Scandinavia) exploring job strain, job control or other job related "stress" exposures were identified. The evidence for an association was assessed according to defined criteria as strong, moderate, limited, or insufficient.

Results There is limited evidence for an association between shift work and stroke, mainly based on results from two occupational cohorts.

There is also limited evidence for high job strain or low job control from cohort studies. Case- crossover studies, which would better reflect short-term effects, were lacking, and the only case-referent study found was very small.

Conclusions There is now fairly solid evidence that shift work and work-related psychosocial stress are risk factors for coronary heart disease; a fact that supports an association also with stroke, another cardiovascular disease. However, the epidemiological evidence for stroke is limited, with few studies, and very limited exposure information. Better study designs are needed to elucidate accumulated as well as triggering/short time effects.

\section{CANCER RISKS AMONG CANADIAN MINING WORKERS IN A POPULATION-BASED COHORT}

1,2Jeavana Sritharan, ${ }^{1} \mathrm{Jill}$ Hardt, ${ }^{1}$ Anna Koné, ${ }^{1,3}$ Anne Harris, ${ }^{2}$ Jasmik Saini, ${ }^{1,2}$ Paul Demers. ${ }^{1}$ Occupational Cancer Research Centre, Cancer Care Ontario, Toronto, Canada;

${ }^{2}$ University of Toronto, Toronto, Canada; ${ }^{3}$ Ryerson University, Toronto, Canada

\subsection{6/oemed-2014-102362.316}

Objectives Mining workers in Canada may be exposed to several potential carcinogens including crystalline silica, various metals, and diesel exhaust. This study aimed to assess the risk of cancer among male mining workers employed in various Canadian mining sectors.

Method The Cohort was created by Statistics Canada through the linkage of the 1991 Canadian Census (long form) to the Canadian Mortality Database, Canadian Cancer Registry, and annual Tax Summary Files (1991-2006). This linkage resulted in a cohort of 1.1 million working males aged $25-74$, including over 14000 workers employed in the mining industry. Cox proportional hazards modelling was used to estimate hazard ratios (HR) and corresponding 95\% confidence intervals, adjusted for age and region.

Results There were 700 cancers among 660 mining industry workers. There was an increased risk for rectal cancer (HR: $1.37,1.01-1.88$ ), particularly in gold mining (HR: 3.11, 1.476.56). Increased risks of kidney and prostate cancer were observed for coal mining (HR: 2.71, 1.12-6.57 and HR: 1.80, 1.10-2.94, respectively), and esophageal cancer in metal mining other than gold or iron (HR: 2.78, 1.13-6.80). There were also elevated risks for stomach and laryngeal cancer among mining workers.
Conclusions This study identified increased risks of rectal, kidney, prostate, and esophageal cancers among male mining industry workers employed in specific sectors. There are also a number of limitations and challenges that accompany the investigation. Our findings may have important implications for our understanding of occupational cancer risk factors and potential policy interventions in the mining industry.

\section{DOSE-TIME-RESPONSE ASSOCIATION BETWEEN OCCUPATIONAL ASBESTOS EXPOSURE AND MESOTHELIOMA}

${ }^{1,2}$ Aude Lacourt, ${ }^{1,2}$ Emilie Lévêque, ${ }^{1,2}{ }^{2}$ aren Leffondré. ${ }^{1}$ INSERM, ISPED, Centre INSERM U897-Epidemiologie-Biostatistique, Bordeaux, France; '2Univ. Bordeaux, ISPED, Centre INSERM U897-Epidemiologie-Biostatistique, Bordeaux, France

\subsection{6/oemed-2014-102362.317}

Objectives In case-control studies on cancer, occupational exposure to a specific agent is often summarised through a cumulative index of exposure at the time of diagnosis/interview. This cumulative index is the sum, over all years of exposure to the agent, of the dose received each year. This gives the same weight to each dose, whether this dose was received in the first years of exposure or at a shorter distant time from the diagnosis/interview. This assumption is unlikely to be reasonable for asbestos and mesothelioma.

The objectives of this study were to estimate the weight of each dose of asbestos received in the past, and to compare the risk of mesothelioma associated with different profiles of exposure, using French case-control data.

Method From a French case-control study, 1199 male cases and 2379 male controls were recruited in 1987-2006. Occupational asbestos exposure was assessed using a job exposure matrix, and represented in logistic regression models by a flexible timedependent weighted function of the dose.

Results The impact of a given increase of the dose depended on when the dose was received. It allowed us to compare the risk of subjects who were exposed for a long duration at a low dose with subjects who were shortly exposed to a high dose at different distant times from diagnosis/interview.

Conclusions This study provides new insights on the dose-timeresponse relationship between occupational asbestos and mesothelioma, and an illustration of the use of an approach that could be of interest for other associations.

\section{COMPARISON OF PERSONAL HABITS AND BASELINE KNOWLEDGE OF HEALTH PROMOTION AMONG STUDENTS OF THE FACULDADE DE MEDICINA DA UNIVERSIDADE DE SÃO PAULO}

Eduardo Sá, Clarissa Medeiros, Renata Arantes, Mario Ferreira Júnior, Lys Rocha. Faculdade de Medicina Da Universidade de São Paulo, São Paulo, Brazil

\subsection{6/oemed-2014-102362.318}

Objectives Because of the importance given to the implementation of health promotion programs nowadays generating positive outcomes important to society and especially to businesses, employees and the financial market this study was designed to assess students' baseline knowledge of expertise in occupational medicine on health promotion and correlate it with the 
students' baseline knowledge Faculdade de Medicina da Universidade de São Paulo (internal fifth-year residents and firstyear internal medicine) as well as evaluate their own health habits.

Method We conducted a cross-sectional study in which we applied two questionnaires that had been used in previous studies by Ferreira Junior, 2011. The questionnaires so called 1) "Survey of attitudes and behaviour" and 2) "Questionnaire assessment of knowledge in health promotion." Regarding the epidemiological data to submit descriptive statistics and Excel filters the table.

Results The higher scores among graduate students, were related to nutrition and oral hygiene, both at present as expected in a year. Questions about professional attitude in their 13 items' scores were higher post-graduate training in the item. In other items, students' grades and graduation from residency and postgraduate students were equal. When comparing the mean of graduate students called in questionnaire 2, all items showed lower values in relation to the notes of interns and residents.

Conclusions The loss in quality of care is undeniable, since there is awareness on the part of the respondents own lack of technical knowledge updated with regard to health promotion that contrasts with the self-perception of adequate training to do so.

\section{CARDIOVASCULAR DISEASE AS A RISK FACTOR FOR DISABILITY RETIREMENT}

Eduardo Sá, Rafael Torres, Lys Rocha. Faculdade de Medicina Da Universidade de São Paulo, São Paulo, Brazil

\subsection{6/oemed-2014-102362.319}

Objectives The relationship between work and some diseases that they can provide is already known for a long time. Because of these conditions some projects were created to improve conditions of work and to support the worker loses her job capacity such, among them the disability retirement. The objective of this study was to identify cardiovascular disease as risk factors for disability retirement.

Method Was realised a literature review, including articles published in 2000 to 2013, being surveyed those in the period from April to November 2013, using the following descriptors: risk factors, pensions and cardiovascular diseases in databases PUBMED/MEDLINE, BIREME, SCOPUS, WEB OF SCIENCE and COCHRANE. Found, respectively, 8, 8, 27, 2 and 0 items. After deleting the duplicate items, those whom were not in English or Portuguese and non revolved around the topic of study, 7 remained. All showed a positive association between disability retirement and cardiovascular diseases.

Results In the study used to compare relative risk for cardiovascular disease retirements with musculoskeletal found the same risk for both diseases. Other studies showed association between increased uric acid, poorly controlled hypertension, perceived stressful work postures and work and increased risk for this retirement. There is a huge investment in prevention campaigns for workers' health to prevent the musculoskeletal disease, but not always the same commitment to the prevention of the cardiovascular.

Conclusions In conclusion cardiovascular disease has high significance for the health of the employee, being an important risk factor for disability retirement, and should be encouraged to implement policies to prevent these.
0295 OCCUPATIONAL EXPOSURE TO CRYSTALLINE SILICA AND THE RISK OF LUNG CANCER IN CANADIAN MEN

1,2 Linda Kachuri, ${ }^{3}$ Paul J Villeneuve, ${ }^{4}$ Marie-Élise Parent, ${ }^{5}$ Kenneth C Johnson, ${ }^{1,2}$ Shelley A Harris. 'Dalla Lana School of Public Health, University of Toronto, Toronto, ON, Canada; '2Prevention and Cancer Control, Cancer Care Ontario, Toronto, ON, Canada; ${ }^{3}$ Department of Health Sciences, Carleton University, Toronto, ON, Canada; ${ }^{4}$ INRS-Institut Armand-Frappier, University of Quebec, Laval, QC, Canada; ${ }^{5}$ Department of Epidemiology and Community Medicine, University of Ottawa, Ottawa, ON, Canada

\subsection{6/oemed-2014-102362.320}

Objectives Crystalline silica is a recognised carcinogen, but the association with lung cancer at lower levels of exposure has not been well characterised. This study investigated the relationship between occupational silica exposure and lung cancer, and the combined effects of cigarette smoking and silica exposure on lung cancer risk.

Method A population-based case-control study was conducted in 8 Canadian provinces between 1994 and 1997. Self-reported questionnaires were used to obtain a lifetime occupational history and information on other risk factors. Occupational hygienists assigned silica exposures to each job based on concentration, frequency, and reliability. Data from 1681 incident lung cancer cases and 2053 controls were analysed using logistic regression to estimate odds ratios (OR) and their 95\% confidence intervals. Models included adjustments for cigarette smoking, lifetime residential second-hand smoke, and occupational exposure to diesel and gasoline engine emissions.

Results Relative to the unexposed, increasing duration of silica exposure at any concentration was associated with a significant trend in lung cancer risk ( $\mathrm{OR} \geq 30$ years: 1.67, 1.21-2.24; ptrend $=0.002$ ). The highest tertile of cumulative silica exposure was associated with lung cancer (OR: 1.81, 1.34-2.42; ptrend $=0.004$ ) relative to the lowest. Men exposed to silica for $\geq 30$ years with $\geq 40$ cigarette pack-years had the highest risk relative to those unexposed with $<10$ pack-years (OR: 42.53, 23.54-76.83). The joint relationship with smoking was consistent with a multiplicative model.

Conclusions Our findings suggest that occupational exposure to silica is a risk factor for lung cancer, independently from active and passive smoking, as well as from exposure to other lung carcinogens.

\section{THE NIEHS GULF STUDY: CORRELATIONS OF CONCENTRATIONS BETWEEN VARIOUS OIL CHEMICALS AND TOTAL HYDROCARBONS}

${ }^{1}$ Caroline Groth, ${ }^{1}$ Sudipto Banerjee, ${ }^{2}$ Tran Huynh, ${ }^{2}$ Gurumurthy Ramachandran, ${ }^{3}$ Mark Stenzel, ${ }^{4}$ Patricia Stewart, ${ }^{5}$ Aaron Blair, ${ }^{6}$ Lawrence Engel, ${ }^{6}$ Dale Sandler, ${ }^{6}$ Richard Kwok. ${ }^{1}$ Division of Biostatistics, University of Minnesota, Minneapolis, MN, USA; ${ }^{2}$ Department of Environmental Health Sciences, University of Minnesota, Minneapolis, MN, USA; ${ }^{3}$ Exposure Assessment Applications, LLC, Arlington, VA, USA; ${ }^{4}$ Stewart Exposure Assessments, LLC, Arlington, VA, USA; ${ }^{5}$ National Cancer Institute, Gaithersburg, MD, USA; ${ }^{6}$ Epidemiology Branch, National Institute of Environmental Health Sciences, Research Triangle Park, NC, USA

\subsection{6/oemed-2014-102362.321}

Objectives In the 2010 Deepwater Horizon Oil Spill, thousands of workers may have been exposed to various potentially harmful chemicals found in crude oil including benzene, ethylbenzene, xylene, and toluene. These and total hydrocarbons (THC) (a composite of all the volatile chemicals in crude oil) were monitored. Over 150000 personal measurements were taken, but many of the measurements of individual chemicals were 